ASME

\section{American Society of Mechanical Engineers}

ASME Accepted Manuscript Repository

Institutional Repository Cover Sheet

ASME Paper Title: Effect of Topological Defects on the Elasticity of Near-Ideal Polymer Networks

Authors: $\quad$ Ghadeer Alamé and Laurence Brassart

ASME Journal Title: Journal of Applied Mechanics

Volume/Issue: $87 / 12$

Date of Publication (VOR* Online): September 16, 2020

https://asmedigitalcollection.asme.org/appliedmechanics/article-

ASME Digital Collection abstract/87/12/121006/1086661/Effect-of-Topological-Defects-on-the-Elasticity-

URL: of?redirectedFrom=fulltext

DOI:

Copyright (C) 2020 by ASME. CC-BY distribution license.

*VOR (version of record) 


\title{
Effect of topological defects on the elasticity of near-ideal polymer networks
}

\author{
Ghadeer Alamé \\ Department of Materials Science \& Engineering \\ Monash University \\ Clayton, VIC 3800, Australia \\ Email: ghadeer.alame@monash.edu \\ Laurence Brassart* \\ Department of Engineering Science \\ University of Oxford \\ Oxford, OX1 3PJ, United Kingdom \\ \& Department of Materials Science \& Engineering \\ Monash University \\ Clayton, VIC 3800, Australia \\ Email: laurence.brassart@eng.ox.ac.uk
}

\begin{abstract}
In recent years, new types of polymer gels have emerged, which have a well-controlled network structure and few topological defects. These so-called near-ideal polymer networks constitute a good model system to revisit the long-standing problem of structure-property relationships in polymer networks, as well as a promising platform for the development of polymer gels with outstanding mechanical properties. In this work, we investigate the relative contributions of network defects (dangling chains and second-order loops) on the stress-stretch response of nearideal polymer networks using a computational discrete network model. We identify the average chain pre-stretch as a key parameter to capture the effect of network topology on the elastic modulus and maximum extensibility. Proper account of the chain pre-stretch further leads to scaling relations for the elastic properties in terms of topology parameters that differ from classical estimates of rubber elasticity theory. Stress-stretch curves calculated using the discrete network model are also compared to semi-analytical estimates.
\end{abstract}

\section{Introduction}

Soft polymeric materials such as elastomers and hydrogels are increasingly being developed for a broad range of applications in engineering and medicine. Recent research efforts have focused on design strategies at the level of the polymer network in order to achieve exceptional mechanical properties in terms of strength, stretchability and toughness [1-4]. However, a quantitative description of the relationships between the topology of the network (including the presence of defects)

${ }^{*}$ Corresponding author. 
and the mechanical properties remains elusive [5]. This is largely due to the fact that networks resulting from conventional random polymerisation and crosslinking processes have an irregular and spatially-heterogeneous structure, which is difficult to characterise and model $[6,7]$.

In recent years, near-ideal polymer networks have been introduced as a new concept for making soft materials with high stiffness and high strength $[1,8,9]$. Near-ideal networks are obtained by the reaction in solution of tetra-arm macromolecules with well-defined arm length. The arms are functionalised with mutually-reactive end groups enabling the cross-coupling of the macromolecules, while preventing two arms of the same macromolecule to react with each other. The resulting networks have been shown to possess a spatially-homogeneous structure with a high conversion degree and essentially no entanglements $[10,11]$. The homogeneity of the network structure is believed to be the reason for the high stiffness and strength of tetra-arm hydrogels [8]. These hydrogels constitute promising model systems to revisit the fundamental question of topology-property relationships in polymer networks.

Unreacted dangling chains and second-order loops are the prevalent topological defects in near-ideal networks prepared near the overlap concentration [11-15]. Dangling chains do not contribute to the elasticity of the network, and effectively lower the coordination of the junctions. Second-order loops are produced when two or more arms of a given four-arm macromolecule react with a corresponding number of arms of another macromolecule. While there have been several attempts to quantify the effect of loops on the mechanical properties, their exact contribution to network elasticity remains poorly understood [15-19].

The objective of this work is to quantify the effects of connectivity defects on the elastic properties of near-ideal polymer networks using a computational random spring network model (Fig. 1). We consider perfect networks of identical freelyjointed chains with fourfold coordination, and introduce defects by altering the junction coordination and by introducing loops. The chain density, average coordination and loop fractions are varied independently to study their relative contributions to the elastic response. While spring network models have been previously used to study the mechanical behaviour of soft materials (see e.g. [20-22] and references therein), to the best of our knowledge they have not been used before to study connectivity defects (including the presence of loops) in 3D networks of freely-jointed chains.

Our results emphasise the role of chain pre-stretch as a key factor to understand topology-property relationships in nearideal networks. For an ideal chain with $N$ Kuhn segments of length $b$, we define the chain pre-stretch as the ratio $R /(\sqrt{N} b)$, where $R$ is the end-to-end distance of a chain in the reference (unstrained) network and $\sqrt{N} b$ is the average end-to-end distance of the corresponding free chain (i.e. the random-walk distance). It can be shown that, for a random network of Gaussian chains with arbitrary topology, the elastic shear modulus of the network is given by (see Appendix A):

$$
G=k T v\left\langle\frac{R^{2}}{N b^{2}}\right\rangle
$$

where $k$ is Boltzmann's constant, $T$ the absolute temperature, and $v$ the density of elastically-effective chains. The notation $\langle\cdot\rangle$ represents the ensemble average over the chains in the network. Eq. (1) shows that topology impacts elasticity via the 
a)

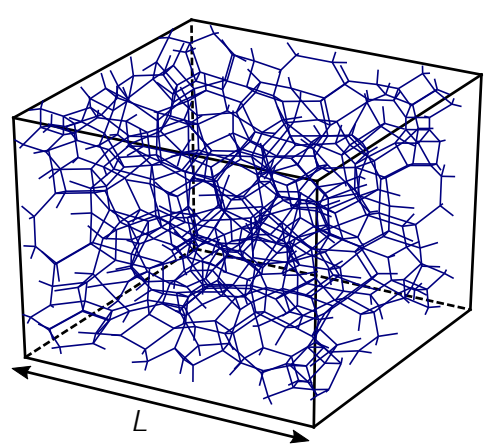

b)

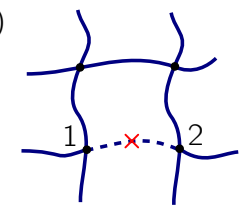

c)

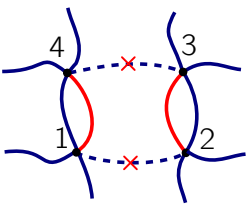

Fig. 1: a) Example of discrete network with 1200 chains. Topological defects are introduced by b) removing chains to reduce the junction coordination, and c) swapping chains between two pairs of adjacent junctions to create second-order loops, without altering coordination.

average pre-stretch only. This result was originally obtained by James [23] and highlighted in recent works [22, 24].

Theories of rubber elasticity commonly assume that chains are all identical, and that their initial end-to-end distance in the unstrained network is the same as that of a free chain, $R=\sqrt{N} b$ [25]. Eq. (1) then reduces to the familiar expression $G=\nu k T$ (affine model). This assumption is reasonable for networks obtained by a random crosslinking process, such as vulcanised rubber, where $N$ must be understood as the average number of Kuhn segments in polymer strands between junctions. In this picture, the average number of Kuhn segments between crosslinks varies with the crosslink density. In contrast, in near-ideal networks formed by cross-coupling well-defined macromolecules in solution, the number of Kuhn segments $N$ is fixed by the molecular weight of the polymer precursors, whereas the initial end-to-end distance of chains in the network depends on polymer concentration and gelation conditions. For example, tetra-arm PEG macromolecules in solution behave as impenetrable spheres, with gyration radius decreasing with concentration [10,26].

In this work we investigate the elastic behaviour of discrete random networks in which the chain pre-stretch is intrinsically coupled to the network structure via the condition of mechanical equilibrium, rather than being specified a priori. The dependence of the chain pre-stretch on the topology parameters leads to scaling relations for the elastic modulus that differ markedly from the classical rubber elasticity theory, but in agreement with Eq. (1). The large-deformation behaviour and limit extensibility are also investigated. We further compare discrete network simulations to results of unit cell-based analytical models, namely the 3-chain model [27], 8-chain model [28], and full-network model [29]. Among those three models, the full-network model gives the most accurate predictions. 


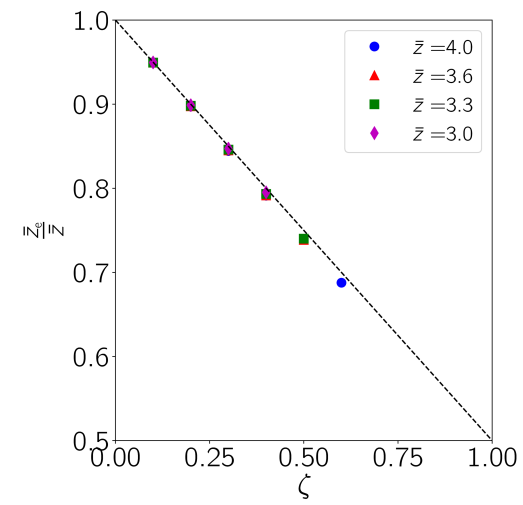

(a)

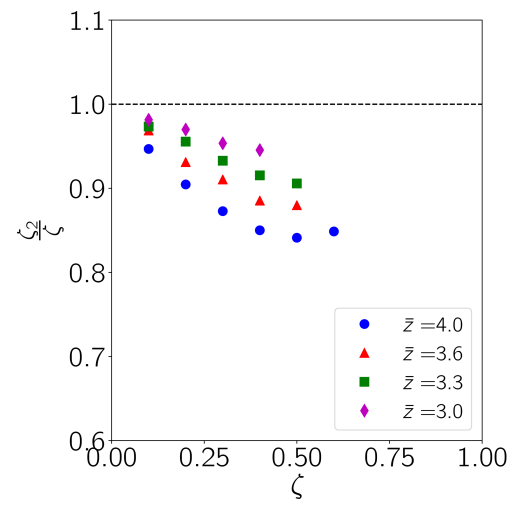

(b)

Fig. 2: a) Ratio of average effective to actual coordination, $\bar{Z}_{e} / \bar{Z}$, as a function of the total loop fraction $\zeta$ for networks with varying topology $(\bar{Z}, \zeta)$. The dashed line represents the approximation $\bar{Z}_{e} / \bar{Z} \approx(1-\zeta / 2)$. b) Relative fraction of double links $\zeta_{2} / \zeta$ as a function of $\zeta$, for networks with varying topology $(\bar{Z}, \zeta)$.

\section{Computational model}

\subsection{Representative network structures}

We consider random Discrete Networks (DN) of $n$ chains connected at $n^{(j)}$ junction points in a volume $V=L^{3}$ (Fig. 1a). The chain density $v$ is defined as the total number of elastically-effective chains $n$ per unit volume:

$$
\mathrm{v}=\frac{n}{L^{3}}
$$

All results presented in the following were obtained using a minimum of 80000 chains. We have conducted convergence studies to verify that the number of chains was sufficiently large. For a given network consisting of $n$ elastically-effective chains and with arbitrary topology, the density is adjusted by changing the length $L$ of the simulation volume, keeping $n$ constant. This approach allows us to vary the density of elastically-effective chains without altering the network topology. The choice of the density of elastically-effective chains as independent parameter in our study is motivated by the consideration that the density of elastically-effective chains is the key network parameter in rubber elasticity theories.

The average coordination number $\bar{Z} \leq 4$ is the average number of chains connected at each junction, $\bar{Z}=n / n^{(j)}$. Perfect networks with $\bar{Z}=4$ were obtained from unstructured, tetrahedral finite element meshes by connecting the centres of neighbouring elements through their common face. Centres of elements adjacent to a boundary were connected to the boundary plane via an additional boundary junction. Meshes were produced using the software GMSH [30], seeding vertices randomly through the volume prior to meshing to ensure randomness and isotropy. Networks with coordination $\bar{Z}<4$ were obtained by randomly cutting links between junctions with a probability $(1-p)$, where $p=1$ corresponds to the perfect network (Fig. 1b). Elastically-ineffective chains and disconnected clusters produced by the chain removal process were removed, as they do not contribute to the elasticity of the network.

Second-order loops were introduced in defected networks in the form of double or triple links, i.e. two or three chains 
connecting the same pair of junctions in parallel. Double links were introduced by randomly swapping links connecting two adjacent pairs of junctions, without altering their coordination (Fig. 1c). The random chain swapping procedure can also produce triple links and quadruple links (the latter form a disconnected cluster). Elastically-ineffective clusters generated by the chain-swapping process were removed from the network, and the final loop fraction was calculated based on the actual number of elastically-effective chains. We define $\zeta_{2}$ (resp. $\zeta_{3}$ ) as the fraction of chains involved in a double (resp. triple) link, and the total loop fraction is given by $\zeta=\zeta_{2}+\zeta_{3}$.

Loops reduce the average number of links per junction point, as compared to a network with the same average coordination but without loops. We define the average effective coordination $\bar{Z}_{e}$ as the average number of links (which include single, double and triple links) per junction in the network, $\bar{Z}_{e}=n^{(l)} / n^{(j)}$. Noting that the number of links and number of chains are related by $n^{(l)}=n\left(1-\zeta_{2} / 2-2 \zeta_{3} / 3\right)$, it follows from the definitions of $\bar{Z}$ and $\bar{Z}_{e}$ that:

$$
\bar{Z}_{e}=\bar{Z}\left(1-(1 / 2) \zeta_{2}-(2 / 3) \zeta_{3}\right)
$$

In the case where $\zeta_{3}$ is small, this relation is well approximated by the simple relation: $\bar{Z}_{e} \approx \bar{Z}\left(1-\frac{\zeta}{2}\right)$. The numerical relationship between $\bar{Z}, \bar{Z}_{e}$ and $\zeta$ is illustrated in Fig. 2a. The relative fraction of double links is illustrated in Fig. 2b, which shows that double links constitute more than $80 \%$ of the loops. The proportion of triple links increases at higher values of $\bar{Z}$, since triple links can only be found between two junctions with a coordination $Z=4$, which become increasingly rare as the average coordination $\bar{Z}$ decreases.

\subsection{Chain behaviour}

We consider networks of identical chains with force-extension behaviour described by the Freely-Jointed Chain (FJC) model. The elastic energy of the chains is given by

$$
w=N k T\left(\frac{r \beta}{N b}+\log \frac{\beta}{\sinh \beta}\right)
$$

where $r$ is the end-to-end distance of the chain and $\beta=\frac{f b}{k T}$ is the normalised force. The force $f$ derives from the elastic energy as $f=\frac{\partial w}{\partial r}$, which gives:

$$
f=\frac{k T}{b} \mathcal{L}^{-1}\left(\frac{r}{N b}\right)
$$

where $\mathcal{L}$ is the Langevin function: $\mathcal{L}(x)=\operatorname{coth}(x)-1 / x$. Throughout this paper, we use $N=100$.

At small chain stretch $(r \lesssim 0.3 \mathrm{Nb})$, the force-extension response is approximately linear and reduces to the Gaussian 
chain model, Eqs (22)-(23) in Appendix A. All networks considered here are such that the large majority of chains are in their Gaussian regime in the reference (unstrained) configuration. A small number of chains pre-stretched beyond their Gaussian regime were found in networks with a large amount of defects (low coordination and/or high fraction of loops).

\subsection{Network behaviour}

Networks are subjected to a prescribed macroscopic deformation gradient tensor $\boldsymbol{F}$, which transforms the initial, cubic volume into a parallelepiped. Accordingly, the position vector $\boldsymbol{x}_{\alpha}$ of each boundary junction $\alpha$ in the current configuration is given by:

$$
\boldsymbol{x}_{\alpha}=\boldsymbol{F} \cdot \boldsymbol{X}_{\alpha}
$$

where $\boldsymbol{X}_{\alpha}$ is the position vector of the junction in the reference (unstrained) configuration. Equilibrium positions of the internal junctions are calculated by minimising the elastic energy for a given $\boldsymbol{F}$. This includes $\boldsymbol{F}=\mathbf{1}$, since networks generated using the procedure described in the previous section are not equilibrated a priori. In practice, we used the algorithm FIRE [31] of the open-source software LAMMPS [32], treating the junctions as atoms and the springs as bonds with user-defined behaviour given by Eq. (5).

In the special case where all chains in the network are in their Gaussian regime (i.e. their force-extension behaviour is linear), it can easily be shown that the displacements of the internal junctions are also affine. This result was previously established by James [23,33] and was recently discussed in [24]. Consider an equilibrated network in the reference configuration, so that the resultant force $f_{\alpha}$ on each internal junction vanishes:

$$
f_{\alpha}=\sum_{\beta \neq \alpha} \kappa \boldsymbol{R}_{\alpha \beta}=\mathbf{0}
$$

where $\boldsymbol{R}_{\alpha \beta} \equiv \boldsymbol{X}_{\beta}-\boldsymbol{X}_{\alpha}$ and $\kappa=3 k T / N b^{2}$ is the stiffness of the Gaussian spring (see Appendix A). The summation extends over all (internal and external) junctions connected to junction $\alpha$ by a spring. We verify that affine displacements $\boldsymbol{x}_{\alpha}=\boldsymbol{F} \cdot \boldsymbol{X}_{\alpha}$ of the internal junctions satisfy the condition of mechanical equilibrium. In the current configuration, the resultant force on an internal junction becomes:

$$
f_{\alpha}=\sum_{\beta \neq \alpha} \kappa r_{\alpha \beta}=\sum_{\beta \neq \alpha} \kappa \boldsymbol{F} \cdot \boldsymbol{R}_{\alpha \beta}=\boldsymbol{F} \cdot \sum_{\beta \neq \alpha} \kappa \boldsymbol{R}_{\alpha \beta}=\mathbf{0}
$$

where $\boldsymbol{r}_{\alpha \beta} \equiv \boldsymbol{x}_{\beta}-\boldsymbol{x}_{\alpha}$. The last equality follows from Eq. (7). We have also used the fact that boundary junctions displace affinely with the macroscopic deformation gradient. Therefore, affine displacement of the internal junctions is the equilib- 
rium solution for a network of Gaussian springs. Affine displacements of internal junctions do not necessarily constitute an equilibrium solution when individual chains are extended beyond their Gaussian regime and the force-extension becomes non-linear. In this case, equilibrium positions of the internal junctions need to be calculated numerically.

Reaction forces at the boundary junctions are used to calculate the macroscopic first Piola-Kirchhoff stress according to (see Appendix B):

$$
\boldsymbol{P}=\frac{1}{V} \sum_{\alpha}\left(\boldsymbol{f}_{\alpha}^{e} \otimes \boldsymbol{X}_{\alpha}\right)
$$

where $f_{\alpha}^{e}$ is the external force vector on junction $\alpha$ and the summation extends over the boundary junctions. The macroscopic Cauchy stress is calculated from the first Piola-Kirchhoff stress by the usual relation: $\boldsymbol{\sigma}=\frac{1}{J} \boldsymbol{P} \cdot \boldsymbol{F}^{T}$, where $J=\operatorname{det}(\boldsymbol{F})$. Since the springs have a zero rest length (cf. Eqs (5) or (22)), networks of freely-jointed chains have vanishing stiffness when subjected to no external force. Networks must therefore be subjected to a hydrostatic stress in their unstrained configuration to prevent network collapse. This hydrostatic pre-stress in turns endow them with initial stiffness.

We consider volume-preserving deformation: $J=1$, and define the macroscopic deformation gradient by the three principal stretches along the edge directions, $\lambda_{1}, \lambda_{2}$ and $\lambda_{3}$. Three loading conditions are considered, namely uniaxial

extension $\left(\lambda_{1} \geq 1, \lambda_{2}=\lambda_{3}=\frac{1}{\sqrt{\lambda_{1}}}\right.$, biaxial extension $\left(\lambda_{1}=\lambda_{2} \geq 1, \lambda_{3}=\frac{1}{\lambda_{1}^{2}}\right)$ and shear $\left(\lambda_{1}=\frac{1}{\lambda_{2}} \geq 1, \lambda_{3}=1\right)$. The three principal Cauchy stresses are written $\sigma_{1}, \sigma_{2}$ and $\sigma_{3}$. Differences in principal stresses are considered in order to eliminate the contribution of the hydrostatic stress required to maintain constant volume. The shear modulus is calculated from the initial slope of the stress-stretch curve in uniaxial extension according to:

$$
3 G=\lim _{\lambda_{1} \rightarrow 1} \frac{\partial\left(\sigma_{1}-\sigma_{3}\right)}{\partial \lambda_{1}} .
$$

At least five realisations of random network structures were considered for each combination of network parameters $v, \bar{Z}$ and $\zeta$. Reported values of the elastic modulus include averages over five random realisations, as well as standard deviations. For the large-deformation response, only one representative stress-stretch curve is represented.

\subsection{Limit extensibility}

Discrete networks were deformed until the numerical simulation failed to find a converged equilibrium solution. This is due to chains being extended close to their contour length, leading to very high forces. We view these events as numerical artefacts, rather than the manifestation of the "true" extensibility limit of an infinitely extended random network with the same topology. As a workaround, we estimated the true extensibility limit $\lambda_{\max }$ by fitting the numerical stress-stretch curves 
with the following expression:

$$
\frac{\sigma_{1}-\sigma_{3}}{G}=\frac{1}{3} \lambda_{\max }\left(\mathcal{L}^{-1}\left(\frac{\lambda_{1}}{\lambda_{\max }}\right) \lambda_{1}-\mathcal{L}^{-1}\left(\frac{\lambda_{3}}{\lambda_{\max }}\right) \lambda_{3}\right) .
$$

Expression (11) is borrowed from the 3-chain model [27] (see also Section 4), with $\lambda_{\max }$ here treated as a fitting parameter corresponding to the value of $\lambda_{1}$ when $\sigma_{1} \rightarrow \infty$.

We also considered a topological estimate $\lambda_{\max }^{S P}$, which relies on the hypothesis that maximum extensibility in uniaxial extension is reached when all the chains in the shortest chain path linking opposite faces of the volume along the tensile direction reach their extensibility limit. The shortest-path estimate of the maximum extensibility is calculated as:

$$
\lambda_{\max }^{S P}=\frac{n^{S P} N b}{L},
$$

where $n^{S P}$ is the number of chains in the shortest chain path. Shortest chain paths were identified by using the Dijkstra algorithm [34] available in the NetworkX Python library. Note that $\lambda_{\max }^{S P}$ is calculated based on the initial network geometry and does not require stretching the network. This also implies that $\lambda_{\max }^{S P}$ is independent of the loading condition. We have verified that the shortest-path estimate $\lambda_{\max }^{S P}$ is very close to the estimate $\lambda_{\max }$ obtained by fitting relation (12) to the stressstretch curves in both uniaxial extension and shear. Note that this is no longer the case in biaxial extension, as in this case extensibility is no longer governed by chain paths linking two opposite faces in the simulation volume. Reported values of maximum extensibility include averages and standard deviations over five random network realisations.

\section{Results}

\subsection{Elastic modulus}

Fig. 3a shows the evolution of the shear modulus $G$ with the density of elastically-effective chains in networks with fixed coordination and no loops. The modulus increases with density as $v^{1 / 3}$ for a fixed value of $\bar{Z}$. The same scaling with density is found in networks containing loops (not shown). On the other hand, the modulus decreases when the average coordination decreases at a fixed value of density (Fig. 3b). For a given value of $\bar{Z}$, the presence of loops further lowers the modulus. The modulus reaches zero when the coordination reaches a critical threshold $\bar{Z}_{c}$, corresponding to the percolation threshold, and which depends on the loop fraction. All the curves collapse onto a single master curve when represented as a function $\bar{Z}-\bar{Z}_{c}$, with $G \sim\left(\bar{Z}-\bar{Z}_{c}\right)^{2 / 3}$ (Fig. 3c).

Chain stretches in the reference network are small, so that the force-extension relationship is approximately linear and Eq. (1) for the modulus holds. According to Eq. (1), network topology affects the elastic modulus of Gaussian networks via the average chain pre-stretch $\bar{R} /(\sqrt{N} b)$, where $\bar{R}=\sqrt{\left\langle R^{2}\right\rangle}$ and $R$ is the end-to-end distance of a chain in the network in the reference configuration. We have verified that the elastic modulus calculated from Eq. (1) using the initial rms- 


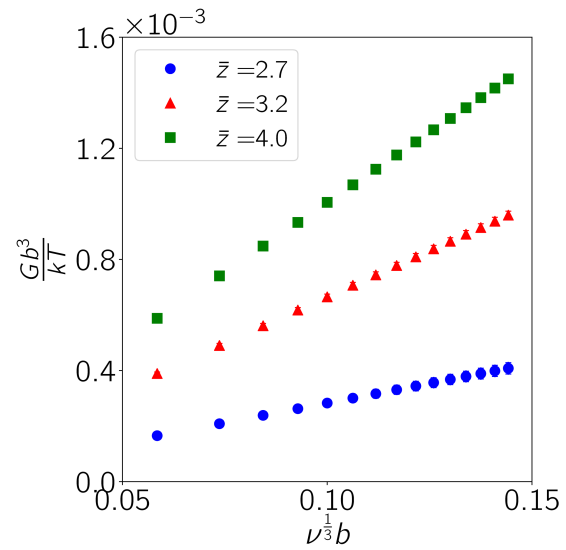

(a)

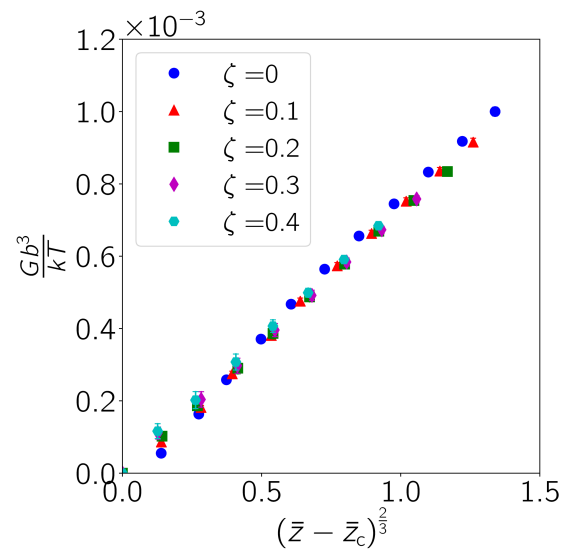

(c)

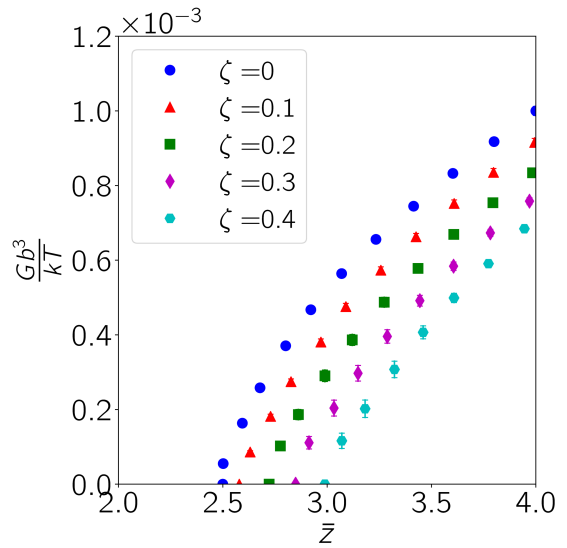

(b)

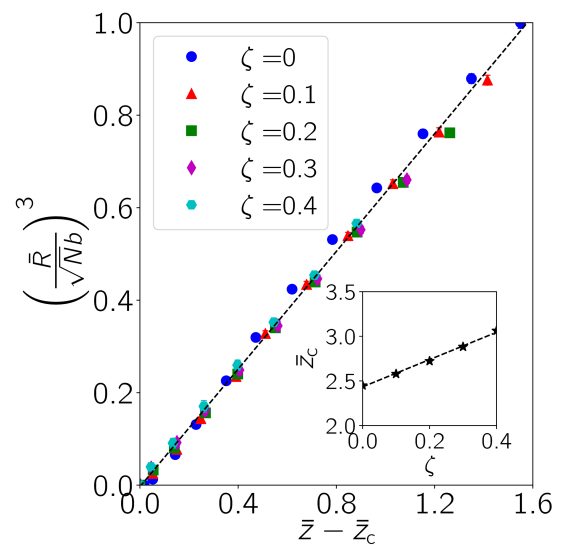

(d)

Fig. 3: a) Evolution of elastic modulus with chain density at a fixed topology $(\bar{Z}, \zeta=0)$. b) Evolution of elastic modulus with average coordination, for various loop fractions and fixed density $v b^{3}=0.001$. c) Curves collapse onto a single master curve when plotted against $\left(\bar{Z}-\bar{Z}_{c}\right)$, where $\bar{Z}_{c}$ varies with the loop fraction. d) Normalised average chain pre-stretch as a function of average coordination, for various loop fractions and fixed density $v b^{3}=0.001$. The dashed line represents the scaling relation (13). The inset shows the scaling of the critical coordination $\bar{Z}_{c}$ with the loop fraction as $\bar{Z}_{c} \approx 1.52 \zeta+2.43$. Symbols represent mean values over five random network realisations, and standard deviations are represented with error bars (barely visible in most cases). The standard deviation on the normalised modulus never exceeds $9 \%$ of the corresponding mean, with the largest standard deviations found at high loop fraction.

average end-to-end distance $\bar{R}$ extracted from the discrete network coincides with the modulus calculated from the slope of the stress-strain response using Eq. (10). Small discrepancies were found in networks with low density or a large amount of defects, due to the presence of chains extended beyond their Gaussian regime. The evolution of average pre-stretch with average coordination is shown in Fig. 3d. As expected, pre-stretch curves also collapse onto a master curve when plotted as a function of $\left(\bar{Z}-\bar{Z}_{c}\right)$. The scaling between the average initial end-to-end distance and network parameters is given by:

$$
\bar{R}^{3} \approx \frac{0.64}{v}\left(\bar{Z}-\bar{Z}_{c}\right)
$$

where $\bar{Z}_{c}$ is a function of loop fraction, with $\bar{Z}_{c} \approx 1.52 \zeta+2.43$. Relation (13) is represented as a dashed line in Fig. $3 \mathrm{~d}$. 
Near $\bar{Z}_{c}$, only a few paths of highly-extended chains span the simulation domain, while the majority of chains have almost collapsed, so that $\bar{R}$ is close to zero.

The scaling relation (13) can be explained based on geometric arguments. $\bar{R}^{3}$ scales as the average volume between junctions, which is of the order of $L^{3} / n^{(j)}$. On the other hand, we have that $L^{3}=n / v$ and $\bar{Z}=n / n^{(j)}$. Combining these relations immediately gives: $\bar{R}^{3} \sim \bar{Z} / \mathrm{v}$, which is consistent with Eq. (13). As $\bar{Z}$ increases at fixed density, the volume between junctions - and thus the chain pre-stretch - also increases in order to keep density constant. On the other hand, increasing the chain density at constant coordination requires compressing the chains by a factor $v^{-1 / 3}$. Finally, it is worth noting that $\bar{R}$ is independent of $N$.

Inserting relation (13) into Eq. (1), the following scaling relation is obtained for the elastic modulus:

$$
\frac{G}{k T} \sim \frac{1}{N b^{2}} v^{1 / 3}\left(\bar{Z}-\bar{Z}_{c}\right)^{2 / 3}
$$

This scaling relation differs markedly from classical scaling relations of rubber elasticity theory (e.g. the affine model, $G \sim v k T)$, where the modulus scales linearly with density and is independent of the number of Kuhn segments. In near-ideal networks, the scaling of the modulus with density is much weaker $\left(\sim v^{1 / 3}\right)$. The weaker scaling is a direct consequence of the coupling between the chain pre-stretch and density. As density increases, the average distance $\bar{R}$ decreases according to Eq. (13), which in turn reduces the ratio $\bar{R} / N b$ and hence the initial chain stiffness, cf. Eq. (22). The softening brought about by the lower pre-stretch partly offsets the increase in free energy arising from increasing the number of chains per unit volume. The scaling with $N^{-1}$ directly follows from the reduced energy in chains with a larger number of Kuhn segments, cf. Eq. (23), together with the fact that $\bar{R}$ is independent of $N$ in the model.

According to Eq. (1), the classical scaling $G \sim v k T$ is recovered when $\bar{R}^{2} \sim N b^{2}$. Accounting for the geometric scaling relation (13), this in turn requires the number of Kuhn segments to vary with density as $N \sim\left(v b^{3}\right)^{-2 / 3}$. We have verified that the classical scaling is indeed recovered when the number of Kuhn segments decreases as chain density increases as indicated. The decrease of $N$ with an increase in density of elastically effective chains is consistent with classical rubber elasticity theory, which considers a vulcanized rubber crosslinked at random junction points. As the crosslink density increases, the average number of Kuhn segments between crosslink points decreases simultaneously, so that on average the chains in the unstrained state have the same end-to-end distance as the corresponding set of free chains [25]. This picture does not hold for near-ideal network where the number of Kuhn segments is independent of chain density.

G-p relation at constant volume The relationships between elastic modulus and connectivity probability $p$ (which dictates the average coordination) has been previously studied in lattices of springs, mostly focusing on percolation phenomena [35-37]. It is well established that Gaussian spring networks belong to a different universality class from Hookean spring networks, but to the same class as the electric conductivity problem [14, 35,38]. Building on this analogy, an effective 
medium approximation was proposed for the elastic modulus as a function of the connectivity probability $[14,39]$ :

$$
\frac{G}{G_{0}} \approx \frac{p-2 / f}{1-2 / f}
$$

where $G_{0}$ is the elastic modulus of the network when $p=1$ and $f$ is the maximum coordination of the junctions. Here, $f=4$ so that $G / G_{0} \approx 2 p-1$. Relationship (15) was obtained assuming that the overall volume occupied by the network remains constant as chains are being cut. This implies that the density of elastically-effective chains changes simultaneously. Fig. 4 shows the normalised modulus $G / G_{0}$ as a function of $p$ for discrete networks with no loops and with a fixed length $L$, together with the effective medium estimate (15). Our computational results agree well with the effective medium estimate far from the percolation threshold $p_{c}$. The latter was calculated as $p_{c} \approx 0.42$, which is close to the theoretical value for a diamond lattice $\left(p_{c} \approx 0.39[14]\right)$. The inset in Fig. 4 shows the response close to the percolation limit, suggesting a critical behaviour of the form $G / G_{0} \sim\left(p-p_{c}\right)^{\gamma}$ with $\gamma \approx 1.87$. The value of the critical exponent is close to the value predicted by de Gennes $(\gamma \approx 1.83)$ [38]. Experimental $G-p$ plots were recently obtained for near-ideal tetra-arm PEG hydrogels [14]. Experimental curves at various concentration of pre-polymers showed good agreement with the theoretical estimate (15) and suggested $\gamma \approx 1.95$, close to our numerical results.

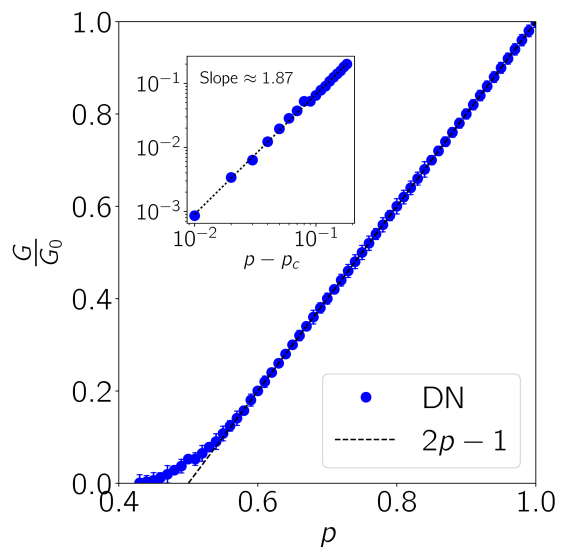

Fig. 4: Evolution of the normalised elastic modulus $G / G_{0}$ with the connectivity probability $p$ at fixed volume $L^{3}$, for $\zeta=0$. The dashed line represents the effective medium estimate $G / G_{0} \approx 2 p-1$ (Eq. 15). The inset illustrates the critical behaviour near the percolation threshold, suggesting $G / G_{0} \sim\left(p-p_{c}\right)^{1.87}$, with $p_{c} \approx 0.42$.

\subsection{Large deformation response}

Stress-stretch curves We consider the large-deformation response of discrete networks in order to determine whether density and topology impact the mechanical response beyond the initial stiffness. Fig. 5 illustrates the relative effects of density, average coordination and loop fraction on the uniaxial extension response. Similar trends were found for the other loading conditions. 


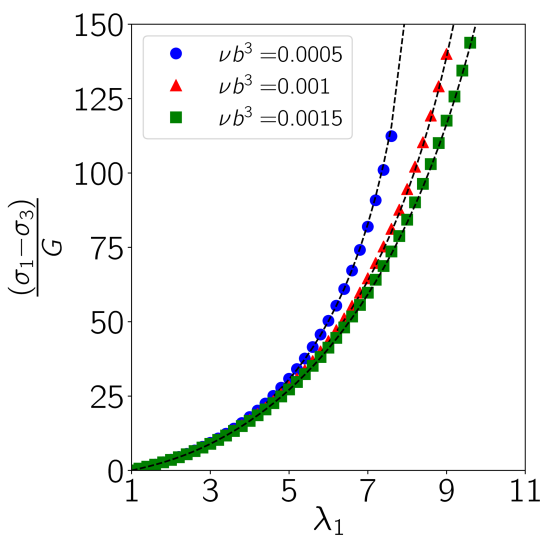

(a)

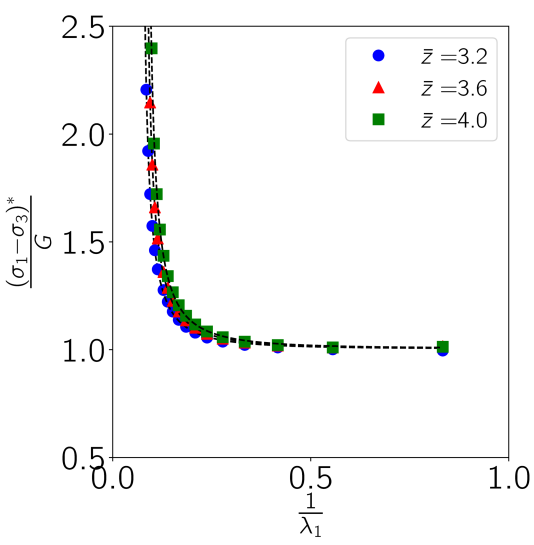

(c)

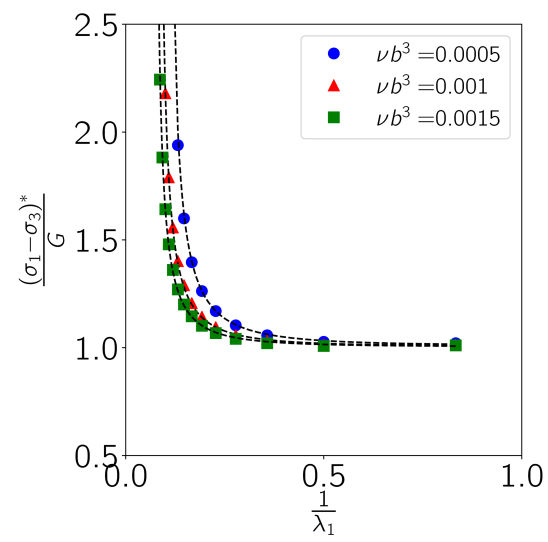

(b)

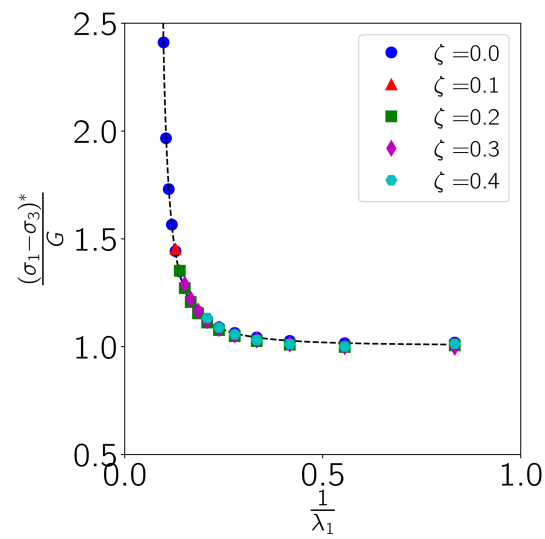

(d)

Fig. 5: a) Normalised uniaxial stress-stretch curves for networks with $\bar{Z}=4$ and $\zeta=0$, for different values of density. b) Normalised Mooney-Rivlin plots of the same data. c) Normalised Mooney-Rivlin plots for networks with fixed density $\left(v b^{3}=0.001\right), \zeta=0$ and different values of average coordination. d) Normalised Mooney-Rivlin plots for networks with fixed density and coordination $\left(v b^{3}=0.001, \bar{Z}=4\right)$ and different loop fractions. The dashed lines represent the fit using relation (11).

Fig. 5a shows the stress-stretch response for different chain densities and fixed topology. Curves are normalised by the initial modulus (which depends on density) so that normalised curves have the same initial slope. Results show that increasing the density reduces the stiffening rate for a given macroscopic stretch, and increases the maximum extensibility. The effect is also seen on the corresponding Mooney-Rivlin plot for the same data in Fig. 5b, which highlights the upward deviation from the Neo-Hookean behaviour at large stretches. In the Mooney-Rivlin plot, we used the notation $\left(\sigma_{1}-\sigma_{3}\right)^{*} \equiv$ $\left(\sigma_{1}-\sigma_{3}\right) /\left(\lambda_{1}^{2}-1 / \lambda_{1}\right)$. Results of Figs $5 \mathrm{a}-5 \mathrm{~b}$ directly follow from the effect of density on the chain pre-stretch, combined to the non-linear response of the chains at large deformation. Chains in denser networks are less pre-stretched and therefore have the capability to be extended more (relative to their initial configuration) before reaching their extensibility limit.

Normalised Mooney-Rivlin plots for varying topology but fixed density are shown in Figs $5 \mathrm{c}$ and $5 \mathrm{~d}$. Overall, the effect of network topology on the normalised stiffening rate is small. Decreasing the average coordination at constant density results in postponing the upward turn in the Mooney plot to larger stretches (Fig. 5c), which is also attributed to the reduction in chain pre-stretch when coordination decreases (Fig. 3d) together with the non-linearity of the FJC model. On the other hand, 
the effect of loops on the large-deformation response is hardly visible on the Mooney-Rivlin plot (Fig. 5d). Note that the presence of loops leads to early failure of the simulations due to local locking effects.

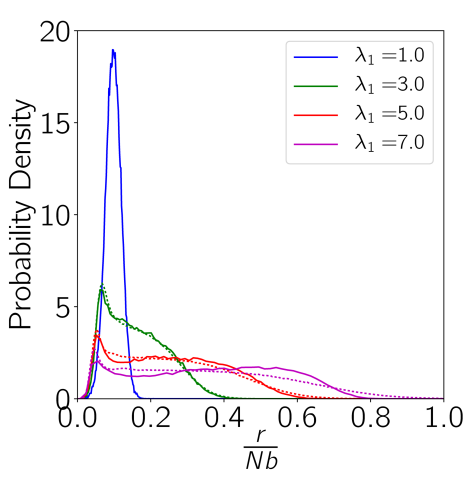

(a)

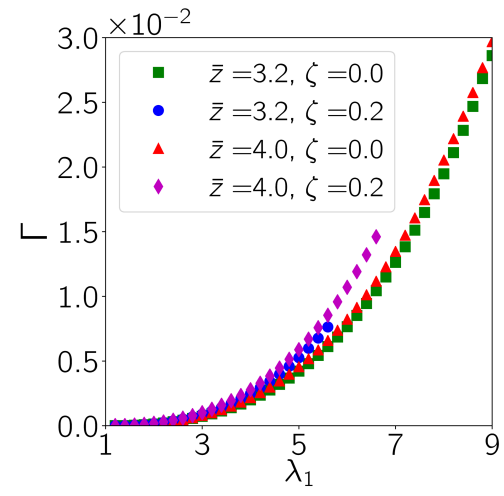

(b)

Fig. 6: a) Probability distributions of chain end-to-end distances at various stretches in equilibrated (continuous lines) and affinely-deformed (dashed lines) perfect networks $(\bar{Z}=4, \zeta=0)$ subjected to uniaxial extension. b) Deviation from affinity as a function of applied stretch in discrete networks for various values of coordination and loop fraction. In this figure, the density was adjusted so that all networks have the same initial average pre-stretch $\bar{R} / \sqrt{N} b=1$.

Affinity We investigate whether chains in the discrete networks deform in an affine manner in the large deformation regime. Let $\boldsymbol{r}_{a f f}$ be the end-to-end vector of a given chain deforming affinely with the applied macroscopic deformation gradient: $\boldsymbol{r}_{a f f}=\boldsymbol{F} \cdot \boldsymbol{R}$, with $\boldsymbol{R}$ the end-to-end vector of the chain in the reference network. Fig. 6a illustrates the probability distribution of chain end-to-end distances in a perfect network $(\bar{Z}=4, \zeta=0)$ subject to uniaxial extension (continuous lines), together with the corresponding distribution obtained assuming that all chains deform affinely (dashed lines). Discrete probability density functions were represented following the procedure proposed in Ref. [40]. The two distributions coincide in the reference configuration by definition, and remain almost identical up to $\lambda \approx 3$. Indeed, it has been shown in Section 2.3 that Gaussian chains in a random network deform in an affine manner. For chains aligned with the main loading direction, departure from the Gaussian regime is expected when $\lambda_{1} R \approx 0.3 N b$ (cf. Section 2.2). For the network considered in Fig. 6a, the average chain pre-stretch is $\bar{R} / \sqrt{N} b \approx 1$ (i.e., $\bar{R} / N b \approx 0.1$ since $N=100$ ) and therefore deviation from affinity is expected when $\lambda_{1} \gtrsim 3$, consistent with the numerical results. In particular, the affinely-deformed network contains a small portion of highly extended chains close to their contour length.

We further quantify deviation from affinity using the following quantity:

$$
\Gamma \equiv \frac{\left\langle r-r_{a f f}\right\rangle}{N b}
$$

where $r=|\boldsymbol{r}|$ and $r_{a f f}=\left|\boldsymbol{r}_{a f f}\right|$. The evolution of $\Gamma$ with the applied stretch is illustrated in Fig. $6 \mathrm{~b}$ for perfect and defected networks having the same average initial pre-stretch $\bar{R} / \sqrt{N} b \approx 1$. The average pre-stretch in defected networks was adjusted 
by varying their density, according to the scaling relation (13). In the figure, curves are interrupted when the DN simulations failed to converge due to the presence of loops, as previously observed in Fig. 5 d. In all cases, $\Gamma \geq 0$, i.e. chains are on average slightly more extended in the equilibrated networks than predicted by the affine assumption. In other words, while affine networks do contain highly extended chains (corresponding to chains initially aligned with the main loading direction), the average end-to-end distance over all the chains is actually higher in the equilibrated networks. In all cases, deviation from affinity remains very small, is essentially independent of network coordination and mildly affected by the presence of loops.

\subsection{Limit extensibility}

The evolution of maximum extensibility $\lambda_{\max }^{S P}$ as a function of density and average coordination is represented in Fig. 7 for uniaxial extension. The maximum extensibility increases as density increases and average coordination decreases. This is again directly related to the change in average chain pre-stretch with these two parameters. As previously mentioned, chains in denser (resp. less-coordinated) networks have a lower pre-stretch in the initial configuration and therefore can accommodate more stretch before reaching their limit extensibility. The role of the chain pre-stretch on maximum extensibility is confirmed by considering the simple estimate $\lambda_{\max } \approx \frac{N b}{\bar{R}}$, which assumes that the limit extensibility is reached when a representative chain with initial end-to-end distance $\bar{R}$ and aligned with the principal loading direction reaches its contour length. Using relation (13), we obtain the following scaling relation in terms of network parameters:

$$
\lambda_{\max } \sim N b v^{1 / 3}\left(\bar{Z}-\bar{Z}_{c}\right)^{-1 / 3}
$$

The scaling relation is represented using dashed lines in Fig. 7. Relation (17) captures the effect of density and average coordination, thus confirming the pre-stretch origin of the roles of these two parameters. In particular, the maximum extensibility tends to infinity when the coordination tends to the critical value $\bar{Z}_{c}$, because the average initial end-to-end distance tends to zero. The approximate relation (17) coincides with the classical estimate $\lambda_{\max }=\sqrt{N}$ in the particular case where $\bar{R}=\sqrt{N} b$. However, it gives markedly different scaling behaviour when $\bar{R}$ is coupled to the network parameters.

The scaling relation (17) fails to predict the effect of loops on the maximum extensibility (Fig. 7b). This is because loops impact the network extensibility in two distinct ways. First, loops reduce the chain pre-stretch at fixed density and coordination (Fig. 3b), which tends to increase the maximum extensibility. Second, loops reduce the shortest chain path. At high coordination values, the second effect dominates, and the presence of loops decreases the maximum extensibility. As coordination decreases and approaches the percolation threshold $\bar{Z}_{c}(\zeta)$, the initial end-to-end distance approaches zero and the maximum extensibility tends to infinity, causing the crossover of curves corresponding to different loop fractions.

\section{Comparison to analytical estimates}

We compare discrete network results to well-known continuum theories of rubber elasticity, namely the 3-chain model [27], 8-chain model [28] and full-network model [29], see also Ref. [41]. 


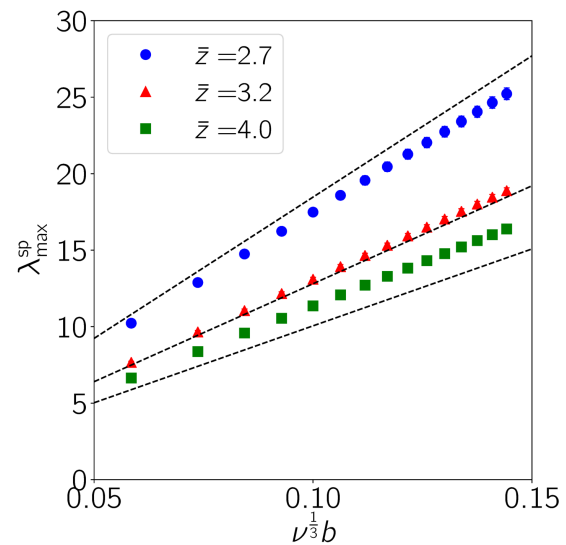

(a)

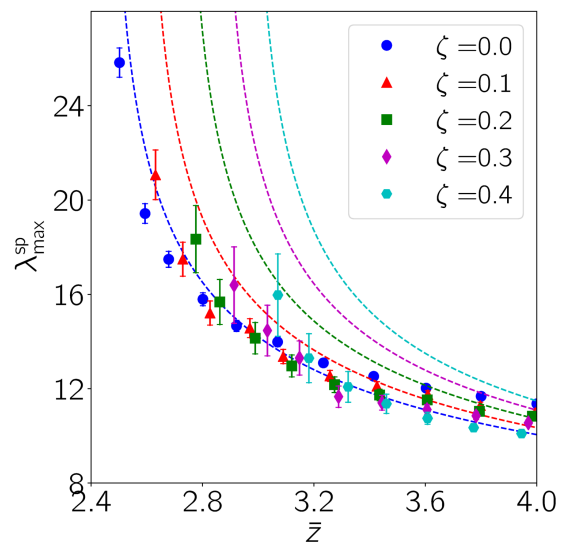

(b)

Fig. 7: a) Evolution of uniaxial maximum extensibility $\lambda_{\max }^{S P}$ with density in networks with fixed coordination $\bar{Z}$ and no loops $(\zeta=0)$. b) Evolution of uniaxial maximum extensibility $\lambda_{\max }^{S P}$ with average coordination in networks with fixed density $v b^{3}=0.001$ and loop fraction $\zeta$. The dashed lines represent the estimate (17).

3-chain model The 3-chain model considers 3 representative chains with initial length $R$ aligned with the 3 principal directions. In the deformed configuration, the chain end-to-end distances become: $r^{(1)}=\lambda_{1} R, r^{(2)}=\lambda_{2} R$ and $r^{(3)}=\lambda_{3} R$. The free energy density is given by $W=(v / 3) \sum_{i=1}^{3} w^{(i)}$, with $w^{(i)}$ the energy of the $i^{t h}$ chain. The stress-stretch relation for volume-preserving deformation is given by:

$$
\frac{\sigma_{1}-\sigma_{3}}{G}=\frac{1}{3} \frac{N b}{R}\left(\mathcal{L}^{-1}\left(\frac{\lambda_{1} R}{N b}\right) \lambda_{1}-\mathcal{L}^{-1}\left(\frac{\lambda_{3} R}{N b}\right) \lambda_{3}\right)
$$

where $G=v k T\left(\frac{R^{2}}{N b^{2}}\right)$, i.e. the affine value (1). Note that we do not a-priori assume that $R=\sqrt{N} b$. This expression for $G$ in Eq. (18) also coincides with Eq. (10) in the case where all chains are in their Gaussian regime in the initial configuration of the network. Maximum extensibility is reached when the maximum principal stretch reaches $N b / R$.

8-chain model The 8-chain model considers 8 representative chains with initial length $R$ connecting each vertex of a cubic unit cell to its centre. The unit cell is aligned with the principal directions. In the deformed configuration, all the chains have the same stretch: $r=\Lambda R$, with $\Lambda=\sqrt{\left(\lambda_{1}^{2}+\lambda_{2}^{2}+\lambda_{3}^{2}\right) / 3}$. The free energy density is given by $W=v w(r)$. The stress-stretch relation is given by:

$$
\frac{\sigma_{1}-\sigma_{3}}{G}=\frac{1}{3} \frac{N b}{R} \frac{1}{\Lambda} \mathcal{L}^{-1}\left(\frac{\Lambda R}{N b}\right)\left(\lambda_{1}^{2}-\lambda_{3}^{2}\right)
$$

where $G$ is the affine modulus (1). Maximum extensibility is reached when $\Lambda=N b / R$. 
Full-network model The full-network model considers a uniform distribution of chain orientations on the unit sphere, with the orientation described by polar and azimuthal angles $(\phi, \theta)$. Chains all have the same initial length $R$ and deform affinely with the macroscopic deformation: $r=\Lambda R$, with

$$
\Lambda=\left(\lambda_{1}^{2} \cos ^{2} \theta \sin ^{2} \phi+\lambda_{2}^{2} \sin ^{2} \theta \sin ^{2} \phi+\lambda_{3}^{2} \cos ^{2} \phi\right)^{1 / 2}
$$

The free energy density is given by $W=v \bar{w}$, where $\bar{w}$ represents the average chain energy over all chain orientations. The stress-stretch relation is given by:

$$
\begin{aligned}
& \frac{\sigma_{1}-\sigma_{3}}{G}=\frac{1}{4 \pi} \frac{N b}{R} \\
& \int_{0}^{2 \pi} \int_{0}^{\pi} \frac{\left(\lambda_{1}^{2} \cos ^{2} \theta \sin ^{3} \phi-\lambda_{3}^{2} \cos ^{2} \phi \sin \phi\right)}{\Lambda} \mathcal{L}^{-1}\left(\frac{\Lambda R}{N b}\right) d \phi d \theta
\end{aligned}
$$

where $G$ is the affine modulus (1). Maximum extensibility is reached when $\Lambda(\phi, \theta)=N b / R$, which corresponds to chains aligned with the direction of maximum principal stretch reaching their contour length. We estimated the integral in Eq. (21) using a 900-points integration scheme $[42,43]$.

The independent input parameters of the analytical models are the Kuhn length $b$, number of Kuhn segments $N$, chain density $v$ and initial chain end-to-end-distance $R$. In practice, these four parameters are often lumped into two effective parameters: the shear modulus $G$ and the maximum chain stretch $N b / R$. However, this practice does not allow to distinguish the roles of individual chain and network parameters on the overall response. In the following, we compare analytical model predictions to discrete networks results using the same set of input parameters. Since the analytical models cannot account for network defects, we consider perfect discrete networks with $\bar{Z}=4$ and $\zeta=0$. We further set the density $v$ such that $\bar{R}=\sqrt{N} b$ in the discrete network, and use $R=\sqrt{N} b$ in the analytical models.

Stress-stretch curves for uniaxial, shear and biaxial loading are shown in Figs 8a-8c. For each loading condition, the initial slopes of the 3-chain, 8-chain, full-network models are identical and coincide with the initial slope of the DN model, which follows from using the same initial average end-to-end distance and density in all the models. Overall, the full-network model provides the best predictions for all three loading conditions, although it slightly underestimates the stiffening in biaxial loading. The 8-chain model consistently underestimates the response. The 3-chain model significantly overestimates the response in uniaxial and shear loading, but gives a reasonable prediction in biaxial loading.

Fig. 9 shows the chain end-to-end distance distributions of the 3-chain, 8-chain, full-network and DN models at different values of the macroscopic stretch $\lambda_{1}$, for the different loading conditions. A Dirac delta distribution arises for the 3-chain and 8-chain models, corresponding to the end-to-end distance of their representative chains. For the full-network model, we considered the end-to-end distances of the 900 chains in the integration scheme over the orientations. The accuracy of the full-network model is correlated to that of its chain end-to-end distance probability density. In particular, the full-network 


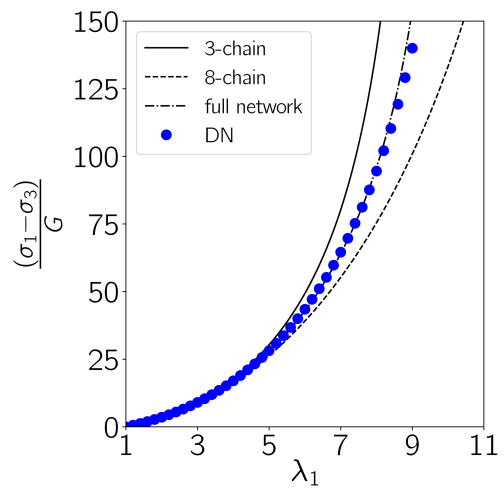

(a)

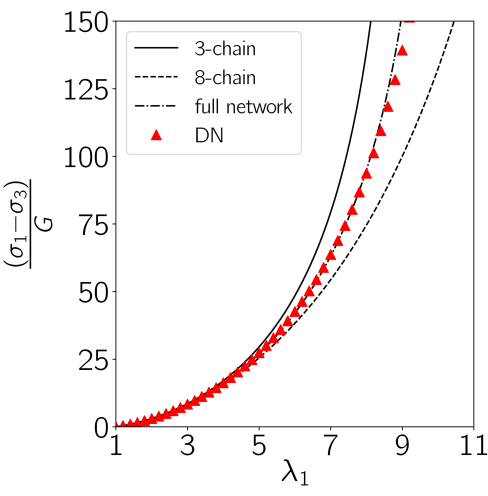

(b)

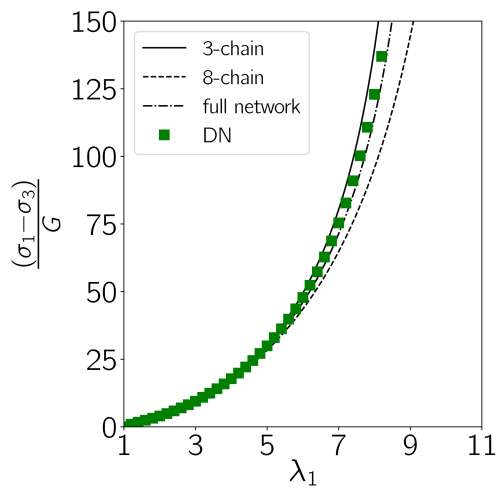

(c)

Fig. 8: Normalised stress-stretch curves based on discrete network (DN) simulations and analytical estimates for a) uniaxial extension; b) shear and c) biaxial extension. DN results correspond to perfect networks with $\bar{Z}=4$ and $\zeta=0$, and the chain density is such that $\bar{R}=\sqrt{N} b$.

model is less able to capture the distribution under biaxial loading (Figs. 9g-9i). The full-network model significantly underestimate the fraction of highly extended chains, which in turns leads to the underestimation in the stress-strain response. This is attributed to the fact that the full-network model considers that all chains have the same end-to-end distance in the unstrained network, whereas the DN model accounts for a distribution of pre-stretch in the unstrained configuration, cf. Fig. 6a for $\lambda_{1}=1$. The 3 -chain model gives a reasonable estimate under biaxial loading because its right peak, corresponding to chains in tension, gives more weigh to the highly-extended chains.

In principle, Relations (18)-(21) can be applied to defected networks as well, accounting for the role of defects via the pre-stretch using the scaling relation (13). The generalisation is valid as far as the elastic modulus is concerned, since the latter depends on topology via the pre-stretch only as established in Eq. (1) (in the Gaussian regime). However, results of Section 3.3 suggest that the role of network defects on maximum extensibility is not full captured by the pre-stretch alone, especially in the presence of loops, cf. Fig. 7.

\section{Conclusions}

We have developed a computational discrete network model to elucidate the effects of network parameters on the elasticity of near-ideal networks. Network parameters include the density of elastically-effective chains, average coordination of the junctions and loop fraction, which were all varied independently. Our results emphasise the role of chain pre-stretch on structure-property relationships in systems where the chain pre-stretch is coupled to network architecture via the conditions of mechanical equilibrium. This notion is very general and could be of importance for a broader class of hydrogel systems. Specific results of our study are the following:

- The network topology affects the elastic modulus through its impact on the average chain pre-stretch. In networks where the average chain pre-stretch is coupled to density and topology, the scaling of the elastic modulus in terms of the network parameters markedly differs from familiar scaling relations of classical rubber elasticity theory.

- Numerical results suggest a scaling relation of the form $G / k T \sim N^{-1} b^{-2} v^{1 / 3}\left(\bar{Z}-\bar{Z}_{c}\right)^{2 / 3}$, where $\bar{Z}_{c}$ is an increasing 


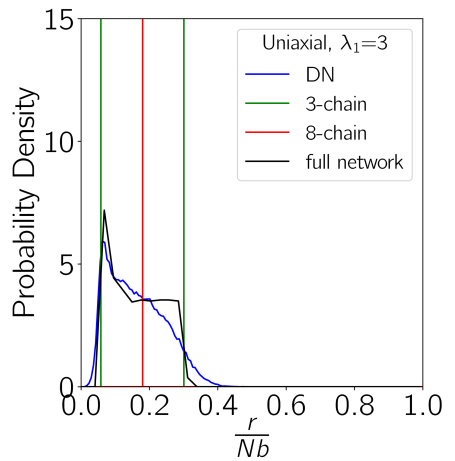

(a)

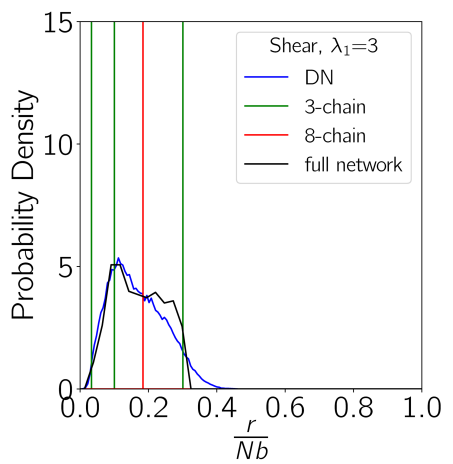

(d)

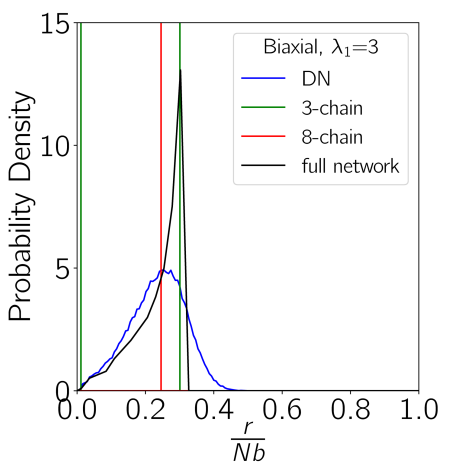

(g)

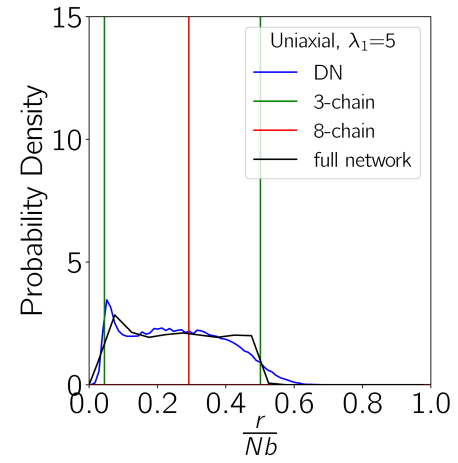

(b)

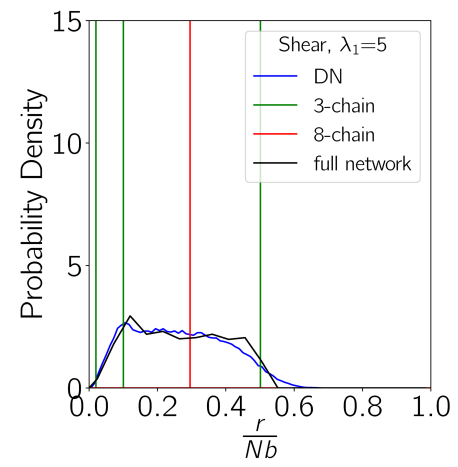

(e)

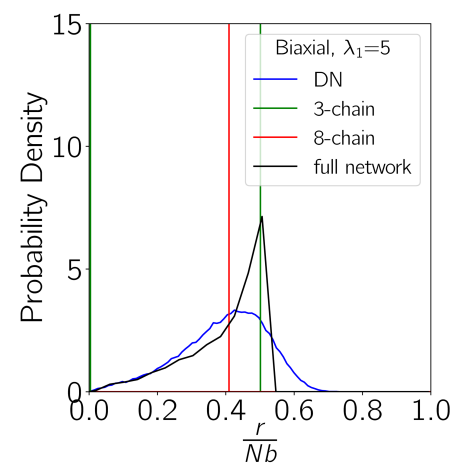

(h)

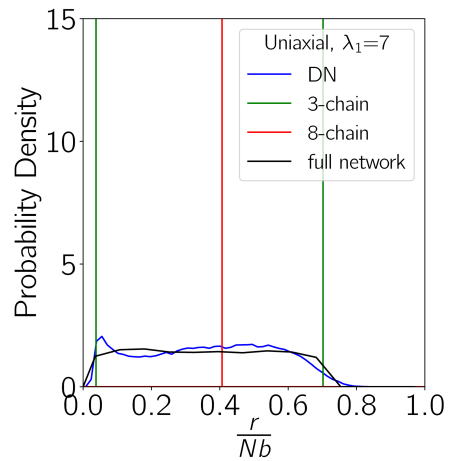

(c)

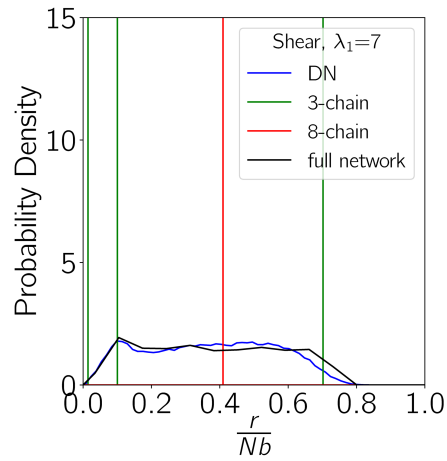

(f)

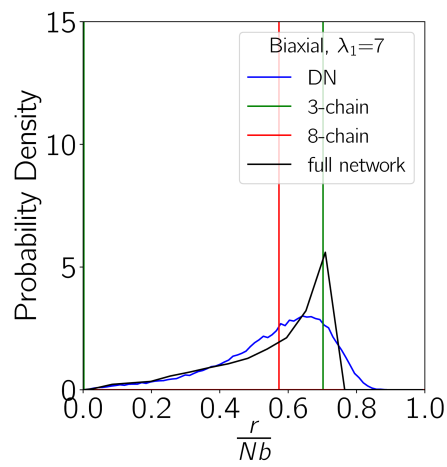

(i)

Fig. 9: Probability distributions of chain end-to-end distances at various stretches in Discrete Networks (DN) and analytical estimates subjected to uniaxial extension (a-c), shear (d-f) and biaxial extension (g-i). Column (a,d,g) correspond to $\lambda_{1}=3$, Column (b,e,h) to $\lambda_{1}=5$ and Column (c,f,i) to $\lambda_{1}=7$. DN results correspond to perfect networks with $\bar{Z}=4$ and $\zeta=0$, and the chain density is such that $\bar{R}=\sqrt{N} b$. Dirac delta distributions arising in the 3-chain and 8-chain models are represented by vertical lines.

function of the loop fraction. The effect of coordination and density on the pre-stretch, and hence on the modulus can be rationalised based on simple geometric arguments together with the affine hypothesis.

- Chains deform in a non-affine manner at large stretches, and deviation from affinity increases in the presence of loops. However, deviation from affinity remains relatively small.

- The limit extensibility also depends primarily on the average chain pre-stretch. At low loop fractions, it is reasonably well described by a simple estimate: $\lambda_{\max } \sim N b v^{1 / 3}\left(\bar{Z}-\bar{Z}_{c}\right)^{-1 / 3}$. Loops further impact the maximum extensibility by 
reducing the shortest chain path via topological changes that are not captured by the average chain pre-stretch.

- Comparison of analytical models to discrete network simulations for perfect networks show that the full-network model

[29] is reasonably accurate under several loading conditions, whereas the 8-chain model systematically underestimates the response. Scaling relations for the chain pre-stretch can be used to generalise classical analytical estimates to cases where $\bar{R} \neq \sqrt{N} b$.

Our results have implications for the interpretation of experimental data for near-ideal polymer networks such as tetra-arm hydrogels. In experiments, the overall chain density is controlled by the concentration of pre-polymer precursors, whereas the density of elastically-effective chains, junction coordination and loop fraction also depend on the reactivity of the arm endgroups and the gelation conditions. Quantitative comparisons between numerical results and experimental results [11,13,14] will be presented in a forthcoming publication.

The proposed computational framework offers many venues for extension. Polydispersity can be readily included by varying the number of Kuhn segments among the chains. Other force-extension relationships, such as the Worm-like chain model, can easily be adopted. Chain scission can also be included, for example using an energy-based criterion [44]. Alternatively, non-permanent crosslinks can also be incorporated, e.g. [21,45]. The computational approach can also be extended to describe double-network gels with either covalent or physical crosslinks, in order to study the role of network architecture as well as chain and crosslink strength on stiffness, strength and toughness [46-48]. In addition to providing detailed understanding of the micro-mechanisms, discrete network models are also promising to generate reference results against which mean-field estimates regarding chain stretch partitioning in rubbers and gels can be assessed, e.g. [49-53].

\section{Acknowledgements}

G. A. is supported by a Monash Graduate Scholarship, and acknowledges the support of the Faculty of Engineering at Monash University for a Graduate Research International Travel Award (GRITA). The authors acknowledge fruitful discussions with Prof. J. Forsythe and Prof. R. P. Jagadeeshan (Monash University).

\section{References}

[1] Sakai, T., Matsunaga, T., Yamamoto, Y., Ito, C., Yoshida, R., Suzuki, S., Sasaki, N., Shibayama, M., and Chung, U.-I., 2008. "Design and fabrication of a high-strength hydrogel with ideally homogeneous network structure from tetrahedron-like macromonomers". Macromol., 41(14), pp. 5379-5384.

[2] Sun, J.-Y., Zhao, X., Illeperuma, W., Chaudhuri, O., Oh, K., Mooney, D., Vlassak, J., and Suo, Z., 2012. "Highly stretchable and tough hydrogels". Nature, 489(7414), pp. 133-136.

[3] Gong, J., 2014. "Materials both tough and soft”. Science, 344(6180), pp. 161-162.

[4] Zhao, X., 2017. "Designing toughness and strength for soft materials". Proc. Natl. Acad. Sci. USA, 114(31), pp. 81388140. 
[5] Yang, C., Yin, T., and Suo, Z., 2019. "Polyacrylamide hydrogels. I. Network imperfection". J. Mech. Phys. Solids, 131, pp. 43-55.

[6] Seiffert, S., 2017. "Origin of nanostructural inhomogeneity in polymer-network gels". Polymer Chemistry, 8, pp. 44724487.

[7] Y., G., Zhao, J., and Johnson, J., 2019. “A (macro)molecular-level understanding of polymer network topology”. Trends Chem., 1(3), pp. 318-334.

[8] Sakai, T., Akagi, Y., Matsunaga, T., Kurakazu, M., Chung, U.-I., and Shibayama, M., 2010. "Highly elastic and deformable hydrogel formed from tetra-arm polymers". Macromol. Rapid Comm., 31(22), pp. $1954-1959$.

[9] Parada, G., and Zhao, X., 2018. "Ideal reversible polymer networks". Soft Matter, 14(25), pp. 5186-5196.

[10] Matsunaga, T., Sakai, T., Akagi, Y., Chung, U.-I., and Shibayama, M., 2009. "SANS and SLS studies on tetra-arm PEG gels in as-prepared and swollen states". Macromolecules, 42, pp. 6245-6252.

[11] Akagi, Y., Gong, J., Chung, U.-i., and Sakai, T., 2013. "Transition between phantom and affine network model observed in polymer gels with controlled network structure". Macromol., 46, pp. 1035-1040.

[12] Lange, F., Schwenke, K., Kurakazu, M., Akagi, Y., Chung, U.-I., Lang, M., Sommer, J.-U., Sakai, T., and Saalwächter, K., 2011. "Connectivity and structural defects in model hydrogels: A combined proton nmr and monte carlo simulation study". Macromol., 44(24), pp. 9666-9674.

[13] Akagi, Y., Katashima, T., Sakurai, H., Chung, U.-i., and Sakai, T., 2013. "Ultimate elongation of polymer gels with controlled network structure". RSC Adv., 3, pp. 13251-13258.

[14] Nishi, K., Fujii, K., Chung, U.-i., Shibayama, M., and Sakai, T., 2017. "Experimental observation of two features unexpected from the classical theories of rubber elasticity". Physical Review Letters, 119, p. 267801.

[15] Lin, T.-S., Wang, R., Johnson, J., and Olsen, B., 2018. "Topological structure of networks formed from symmetric four-arm precursors”. Macromolecules, 51(3), pp. 1224-1231.

[16] Zhang, A., Wang, R., Kawamoto, K., Olsen, B., and Johnson, J., 2016. "Quantifying the impact of molecular defects on polymer network elasticity”. Science, 353(6305), pp. 1264-1268.

[17] Lin, T.-S., Wang, R., Johnson, J., and Olsen, B., 2018. "Revisiting the elasticity theory for real gaussian phantom networks". Macromolecules, 52, pp. 1685-1694.

[18] Panyukov, S., 2019. “Loops in polymer networks”. Macromol., 52(11), pp. 4145-4153.

[19] Lang, M., 2019. “On the elasticity of polymer model networks containing finite loops”. Macromol., 52(16), pp. 62666273.

[20] Sugimura, A., Makoto, A., Matsunaga, T., Akagi, Y., Sakai, T. Noguchi, H., and Shibayama, M., 2013. "Mechanical properties of a polymer network of tetra-peg gel”. Polym. J., 45, pp. 300-306.

[21] Kothari, K., Yuhang, H., Gupta, S., and Elbanna, A., 2018. "Mechanical response of two-dimensional polymer networks: Role of topology, rate dependence, and damage accumulation”. J. Appl. Mech., 85(3), p. 031008.

[22] Alamé, G., and Brassart, L., 2019. "Relative contributions of chain density and topology to the elasticity of twodimensional polymer networks". Soft Matter, 15, pp. 5703-5713. 
[23] James, H., and Guth, E., 1947. “Theory of the elastic properties of rubber”. J. Chem. Phys., 11(10), pp. $455-481$.

[24] Gusev, A., 2019. "Numerical estimates of the topological effects in the elasticity of gaussian polymer networks and their exact theoretical description". Macromolecules, 52, pp. 3244-3251.

[25] Treloar, L., 1975. The physics of rubber elasticity. Oxford University Press.

[26] Akagi, Y., Matsunaga, T., Shibayama, M., Chung, U.-i., and Sakai, T., 2010. "Evaluation of topological defects in tetra-peg gels". Macromol., 43, pp. 488-493.

[27] Wang, M., and Guth, E., 1952. "Statistical theory of networks of non-gaussian flexible chains". The Journal of Chemical Physics, 20(7), pp. 1144-1157.

[28] Arruda, E., and Boyce, M., 1993. "A three-dimensional constitutive model for the large stretch behavior of rubber elastic materials". Journal of the Mechanics and Physics of Solids, 41, pp. 389-412.

[29] Wu, P., and Van Der Giessen, E., 1993. “On improved network models for rubber elasticity and their applications to orientation hardening in glassy polymers". Journal of the Mechanics and Physics of Solids, 41(3), pp. 427-456.

[30] Geuzaine, C., and Remacle, J.-F., 2009. "Gmsh: A 3-D finite element mesh generator with built-in pre- and postprocessing facilities". Int. J. Numer. Meth. Eng., 79, pp. 1309-1331.

[31] Bitzek, E., Koskinen, P., Gähler, F., Moseler, M., and Gumbsch, P., 2006. "Structural relaxation made simple”. Physical review letters, 97(17), p. 170201

[32] Plimpton, S., 1995. "Fast parallel algorithms for short-range molecular dynamics". J. Comput. Phys., 117, pp. 1-19.

[33] James, H., 1947. "Statistical properties of networks of flexible chains”. J. Chem. Phys., 15(9), pp. 651-668.

[34] Dijkstra, E., 1959. "A note on two problems in connexion with graphs”. Numer. Math., 1(1), pp. $269-271$.

[35] Feng, S., and Sen, P., 1984. "Percolation on elastic networks: new exponent and threshold". Phys. Rev. Lett., 52(3), pp. 216-219.

[36] Tang, W., and Thorpe, M., 1987. "Mapping between random central-force networks and random resistor networks". Phys. Rev. B, 36(7), pp. 3798-3804.

[37] Arbabi, S., and Sahimi, M., 1993. "Mechanics of disordered solids. i. percolation on elastic networks with central forces". Phys. Rev. B, 47(2), pp. 695-702.

[38] De Gennes, P., 1976. “On a relation between percolation theory and the elasticity of gels”. J. Phys. Lett., 37(1), pp. 1-2.

[39] Nishi, K., Chijishi, M., Katsumo, Y., Nakao, T., Fujii, K., Chung, U.-i., Nogushi, H., Sakai, T., and Shibayama, M., 2012. "Rubber elasticity for incomplete polymer networks". J. Chem. Phys., 137(22), p. 224903.

[40] Scott, D. W., 1979. “On optimal and data-based histograms”. Biometrika, 66(3), pp. 605-610.

[41] Boyce, M., and Arruda, E., 2000. "Constitutive models of rubber elasticity: A review”. Rubber Chem. Technol., 73, pp. 504-523.

[42] Fliege, J., and Maier, U., 1999. "The distribution of points on the sphere and corresponding cubature formulae". IMA Journal of Numerical Analysis, 19(2), pp. 317-334.

[43] Itskov, M., 2016. "On the accuracy of numerical integration over the unit sphere applied to full network models". Computational Mechanics, 57(5), pp. 859-865. 
[44] Mao, Y., Talamini, B., and Anand, L., 2017. "Rupture of polymers by chain scission". Extreme Mech. Lett., 13, pp. 17-24.

[45] Iyer, B., Yashin, V., Hamer, M., Kowalewski, T., Matyjaszewski, K., and Balazs, A., 2015. "Ductility, toughness and strain recovery in self-healing dual cross-linked nanoparticle networks studied by computer simulations". Prog. Polym. Sci., 40, pp. 121-137.

[46] Wang, X., and Hong, W., 2011. "Pseudo-elasticity of a double network gel”. Soft Matter, 7, pp. 8576-8581.

[47] Zhao, X., 2012. "A theory for large deformation and damage of interpenetrating polymer networks". J. Mech. Phys. Solids, 60, pp. 319-332.

[48] Xin, H., Saricilar, S., Brown, H., Whitten, P., and Spinks, G., 2013. "Effect of first network topology on the toughness of double network hydrogels". Macromol., 46, pp. 6613-6620.

[49] Miehe, C., Göktepe, S., and Lulei, F., 2004. "A micro-macro approach to rubber-like materials - part i: the non-affine micro-sphere model of rubber elasticity". J. Mech. Phys. Solids, 52, pp. 2617-2660.

[50] Itskov, M., and Knyazeva, A., 2016. "A rubber elasticity and softening model based on chain length statistics". Int. J. Solids Struct., 80, pp. 512-519.

[51] Cioroianu, A., Spiesz, E., and Storm, C., 2016. "Disorder, pre-stress and non-affinity in polymer 8-chain models". J. Mech. Phys. Solids, 89, pp. 110-125.

[52] Verron, E., and Gros, A., 2017. "An equal force theory for network models of soft materials with arbitrary molecular weight distribution". J. Mech. Phys. Solids, 106, pp. 176-190.

[53] Tehrani, M., and Sarvestani, A., 2017. "Effect of chain length distribution on mechanical behavior of polymeric networks". Eur. Polym. J., 87, pp. 136-146.

[54] Hill, R., 1967. "The essential structure of constitutive laws for metal composites and polycrystals". Journal of the Mechanics and Physics of Solids, 15, pp. 79-95.

[55] Blanco, P., Sànchez, P., de Souza Neto, E., and Feijóo, R., 2016. "Variational foundations and generalized unified theory of rve-based multiscale models". Archives in Computational Methods in Engineering, 23, pp. $191-253$.

\section{Appendix A: Elastic modulus of random Gaussian spring networks}

Consider a random network of identical springs with behaviour described by the Gaussian chain model:

$$
f=3 k T \frac{r}{N b^{2}}
$$


This relation corresponds to the the small force limit of the freely-jointed chain model (5). The elastic energy of each spring is thus given by:

$$
w=\frac{3}{2} k T \frac{r^{2}}{N b^{2}} .
$$

The elastic energy density of the network (energy per unit volume in the reference configuration) is obtained by summing the elastic energy of all the chains:

$$
W=\frac{1}{V} \frac{3}{2} \frac{k T}{N b^{2}} \sum_{n}\left(r^{(i)}\right)^{2},
$$

with $\boldsymbol{r}^{(i)}$ the end-to-end vector of the $i^{\text {th }}$ spring in the network and $r^{(i)}=\left|\boldsymbol{r}^{(i)}\right|$. We write $\boldsymbol{R}^{(i)}$ the corresponding end-to-end vectors in the reference (unstrained) configuration.

It is shown in Section 2.3 that the equilibrium displacements of internal junctions in a network of Gaussian springs subjected to affine displacements of its external junctions are also affine. In this case, the energy (24) becomes:

$$
W=\frac{1}{V} \frac{3}{2} \frac{k T}{N b^{2}} \sum^{n}\left(\lambda_{1}^{2}\left(R_{1}^{(i)}\right)^{2}+\lambda_{2}^{2}\left(R_{2}^{(i)}\right)^{2}+\lambda_{3}^{2}\left(R_{3}^{(i)}\right)^{2}\right)
$$

where $R_{i}^{(i)}(i=1,2,3)$ are the three components of the initial end-to-end vector between two junction points. If the springs are randomly oriented, the expression simplifies as [25]:

$$
W=\frac{G}{2}\left(\lambda_{1}^{2}+\lambda_{2}^{2}+\lambda_{3}^{2}\right)
$$

where the shear modulus $G$ is given by:

$$
G=k T \vee \frac{\bar{R}^{2}}{N b^{2}},
$$

with $\bar{R}^{2} \equiv\left\langle R^{2}\right\rangle=\frac{1}{n} \sum_{n}\left(R^{(i)}\right)^{2}$ and $v=n / V$. In the particular case where $\bar{R}=\sqrt{N} b$, Eq. (27) recovers the familiar affine estimate of rubber elasticity theory: $G=v k T$ [25]. In general however, the value $\bar{R}^{2}$ depends on density and topology, and $\bar{R} \neq \sqrt{N} b$. Expression (27) is exact, provided that the springs are randomly oriented and obey relation (22). 


\section{Appendix B: Macroscopic stress of random networks}

We derive an expression for the macroscopic first Piola-Kirchhoff stress by requiring that the work of the macroscopic stress $\boldsymbol{P}$ in a (virtual) deformation $\delta \boldsymbol{F}$ should be equal to the work of the external forces in displacements $\delta \boldsymbol{r}_{\alpha}$ compatible with $\delta \boldsymbol{F}$ :

$$
V P: \delta F=\sum_{\alpha} f_{\alpha}^{e} \cdot \delta x_{\alpha}
$$

where $f_{\alpha}^{e}$ is the reaction force acting on junction $\alpha$ and the summation extends over all the boundary junctions. Relation (28) ensures energy consistency from the micro to the macro scale, as a fundamental requirement in RVE-based homogenisation problems [54,55]. Using Eq. (6), the right-hand side of Eq. (28) rewrites as:

$$
\sum_{\alpha} \boldsymbol{f}_{\alpha}^{e} \cdot \delta \boldsymbol{x}_{\alpha}=\sum_{\alpha} \boldsymbol{f}_{\alpha}^{e} \cdot\left(\delta \boldsymbol{F} \cdot \boldsymbol{X}_{\alpha}\right)=\sum_{\alpha}\left(\boldsymbol{f}_{\alpha}^{e} \otimes \boldsymbol{X}_{\alpha}\right): \delta \boldsymbol{F}
$$

Comparing Eqs (28) and (29), the macroscopic stress is identified as:

$$
\boldsymbol{P}=\frac{1}{V} \sum_{\alpha}\left(\boldsymbol{f}_{\alpha}^{e} \otimes \boldsymbol{X}_{\alpha}\right)
$$

It can be verified that the macroscopic stress defined by (30) coincides with $\partial W / \partial \boldsymbol{F}$, where $W$ is the macroscopic elastic energy (i.e. the sum of the elastic energies of all the chains in the network). 